\title{
PLANAR TRAVELLING WAVES IN INCOMPRESSIBLE ELASTIC RODS*
}

\author{
STUART S. ANTMAN ${ }^{\dagger}$ AND GREGORY M. CROSSWHITE ${ }^{\ddagger}$
}

This paper is dedicated to George Papanicolaou on the occasion of his 60th birthday

\begin{abstract}
This paper first describes the rich and intricate structure of the quasilinear hyperbolic equations governing the planar motions of incompressible nonlinearly elastic rods. It then shows that under a variety of constitutive restrictions, these equations have periodic travelling-wave solutions coupling longitudinal and transverse extension, flexure, and shear.
\end{abstract}

Key words. incompressible nonlinearly elastic rods, travelling waves

AMS subject classifications. 74K10, 74B20, 35B35

1. Introduction. Travelling waves for nonlinearly elastic structures form a rich collection of exact solutions to the governing quasilinear hyperbolic equations (see [3]). (Travelling waves for such structures with viscous dissipation, not treated here, give important information about the shock structure of the corresponding system without dissipation.) In this paper we study planar travelling waves for naturally straight incompressible nonlinearly elastic rods. The governing equations have a mathematical structure strikingly different from that for compressible rods [3], whose equations are completely integrable. Our aim here is not to supply a comprehensive treatment of travelling waves, but merely to give a taste of the physical and mathematical richness of the equations.

There have been a number of interesting studies, e.g., [5, 16], of purely extensional motions of incompressible nonlinear elastic rods, which lead to the analyses of phase portraits. Here we emphasize more complicated motions in which flexure and shear are coupled with longitudinal and tranvsverse extension.

Since we limit our attention to elastic rods, we can formulate their equations of motion as the Euler-Lagrange equations for the Lagrangian (action) functional. We immediately specialize these equations to those for travelling waves, noting that the structure of their Lagrangian is quite different from that for the dynamics of systems of rigid bodies.

We then discuss cases in which our travelling-wave equations can be put into Hamiltonian form and in which this Hamiltonian form has attractive properties. For these cases we show how some of the extensive qualitative theory of Hamiltonian ordinary differential equations implies that under various constitutive assumptions there are periodic travelling waves.

Notation. Vectors, which are elements of Euclidean 3-space $\mathbb{E}^{3}$, and vector-valued functions are denoted by lower-case, italic, bold-face symbols. The dot product and cross product of (vectors) $\boldsymbol{u}$ and $\boldsymbol{v}$ are denoted $\boldsymbol{u} \cdot \boldsymbol{v}$ and $\boldsymbol{u} \times \boldsymbol{v}$.

The (Gâteaux) differential of the function $\boldsymbol{u} \mapsto \boldsymbol{f}(\boldsymbol{u})$ at $\boldsymbol{v}$ in the direction $\boldsymbol{h}$ is $\left.\frac{d}{d t} \boldsymbol{f}(\boldsymbol{v}+t \boldsymbol{h})\right|_{t=0}$. When it is linear in $\boldsymbol{h}$, we denote this differential by $\frac{\partial f}{\partial \boldsymbol{u}}(\boldsymbol{v}) \cdot \boldsymbol{h}$

\footnotetext{
*Received August 31, 2004; accepted for publication December 3, 2004.

${ }^{\dagger}$ Department of Mathematics, Institutes for Physical Science and Technology and for Systems Research, University of Maryland, College Park, MD 20742-4015, U.S.A. (ssa@math.umd.edu).

$\ddagger$ Departments of Mathematics, Physics, and Computer Science, University of Maryland, College Park, MD 20742-4015, U.S.A. (cog@wam.umd.edu).
} 
or $\boldsymbol{f}_{\boldsymbol{u}}(\boldsymbol{v}) \cdot \boldsymbol{h}$. We occasionally denote the function $\boldsymbol{u} \mapsto \boldsymbol{f}(\boldsymbol{u})$ by $\boldsymbol{f}(\cdot)$. The partial derivative of a function $f$ with respect to the scalar argument $t$ is denoted by either $f_{t}$ or $\partial_{t} f$. The operator $\partial_{t}$ is assumed to apply only to the term immediately following it. We shall always use notation like $\partial_{t}$ for a total derivative, i.e., a derivative of a composite function. Obvious analogs of these notations will also be used.

The symbol $C$, occasionally bearing a subscript, always denotes a positive constant that is supplied as data or that can be estimated in terms of data. Its meaning usually changes with each appearance (even in the same equation or inequality).

2. Formulation of the Governing Equations. We outline the formulation of virtually the only geometrically exact theory for the planar deformation of incompressible rods that leads to local equations in which Lagrange multipliers do not intervene. For details, see $[1,2,4]$.

Kinematics of deformation. Let $\{\boldsymbol{i}, \boldsymbol{j}, \boldsymbol{k}\}$ be a right-handed orthonormal basis for Euclidean 3-space. Let $h$ be a small positive number. We take the reference configuration of a planar rod-like body to be the infinite strip $\{s \boldsymbol{i}+\xi \boldsymbol{j}: s \in \mathbb{R},|\xi| \leq h\}$. The material line $s \mapsto s \boldsymbol{i}$ in $\mathcal{B}$ is the axis.

Let

$$
\boldsymbol{a}(\theta):=\cos \theta \boldsymbol{i}+\sin \theta \boldsymbol{j}, \quad \boldsymbol{b}(\theta):=-\sin \theta \boldsymbol{i}+\cos \theta \boldsymbol{j} .
$$

We constrain the position $\boldsymbol{p}(s, \xi, t)$ of material point $s \boldsymbol{i}+\xi \boldsymbol{j}$ at time $t$ to be determined by the values of two functions $(s, t) \mapsto \boldsymbol{r}(s, t) \in \operatorname{span}\{\boldsymbol{i}, \boldsymbol{j}\}$ and $(s, t) \mapsto \theta(s, t) \in \mathbb{R}$ according to

$$
\boldsymbol{p}(s, \xi, t)=\boldsymbol{r}(s, t)+\beta\left(\boldsymbol{r}_{s}(s, t) \cdot \boldsymbol{a}(\theta(s, t)), \theta_{s}(s, t), \xi\right) \boldsymbol{b}(\theta(s, t))
$$

where

$$
\beta(\nu, \mu, \xi):=\frac{2 \xi}{\nu+\delta(\nu, \mu, \xi)} \equiv \frac{\nu-\delta(\nu, \mu, \xi)}{\mu}, \quad \delta(\nu, \mu, \xi):=\sqrt{\nu^{2}-2 \xi \mu} .
$$

The form of $\beta$, which ensures that the Jacobian of $\boldsymbol{p}(\cdot, \cdot, t)$ is identically 1 , is found as the solution of the equation for $\gamma$ obtained by the requirement that the constrained position field $\boldsymbol{p}=\boldsymbol{r}+\gamma \boldsymbol{b}$ have Jacobian $1[1,2,4]$.

We define the strain variables $\nu, \eta, \mu$ by

$$
\boldsymbol{r}_{s}=: \nu \boldsymbol{a}+\eta \boldsymbol{b}, \quad \mu:=\theta_{s} .
$$

The strain $\nu=\boldsymbol{k} \cdot\left(\boldsymbol{r}_{s} \times \boldsymbol{b}\right)$ is the (ratio of the) area of the parallelogram with sides $\boldsymbol{r}_{s}$ and $\boldsymbol{b}$ (to the area 1 of its pre-image in the reference configuration). It effectively measures elongation. The shear strain $\eta=\boldsymbol{r}_{s} \cdot \boldsymbol{b}=:\left|\boldsymbol{r}_{s}\right| \sin \left(\frac{\pi}{2}-\gamma\right)$ measures the reduction $\gamma$ in angle between $\boldsymbol{b}$ and the tangent $\boldsymbol{r}_{s} /\left|\boldsymbol{r}_{s}\right|$. The strain $\mu$ measures the amount of bending. (It is not a curvature because $\theta$ is not the tangent angle to $r$ and because $s$ is not the arc length of the deformed axis.) We get a theory for unshearable rods, not treated here, by constraining $\eta=0$. In this case, $\nu=\left|\boldsymbol{r}_{s}\right|$ and $\boldsymbol{b}=\boldsymbol{k} \times \boldsymbol{r}_{s} / \nu$.

From (2.2) and (2.4) we get

$$
\begin{gathered}
\boldsymbol{p}_{s}=\boldsymbol{r}_{s}+\left(\beta_{\nu} \nu_{s}+\beta_{\mu} \mu_{s}\right) \boldsymbol{b}-\beta \mu \boldsymbol{a}, \quad \boldsymbol{p}_{\xi}=\beta_{\xi} \boldsymbol{b}, \\
\boldsymbol{p}_{t}=\boldsymbol{r}_{t}+\left(\beta_{\nu} \nu_{t}+\beta_{\mu} \mu_{t}\right) \boldsymbol{b}-\beta \theta_{t} \boldsymbol{a}
\end{gathered}
$$


with

$$
\begin{gathered}
\delta_{\nu}=\frac{\nu}{\delta}, \quad \delta_{\mu}=-\frac{\xi}{\delta}, \quad \delta_{\xi}=-\frac{\mu}{\delta} \\
\beta_{\nu}=-\frac{2 \xi}{\delta(\nu+\delta)}=-\frac{\beta}{\delta}, \quad \beta_{\mu}=\frac{2 \xi^{2}}{\delta(\nu+\delta)^{2}}=\frac{\beta^{2}}{2 \delta}, \quad \beta_{\xi}=\frac{1}{\delta}
\end{gathered}
$$

We let $s$ correspond to index 1 and $\xi$ to index 2. Then (2.5) implies that the components of the right Cauchy-Green deformation tensor $\boldsymbol{C}$ are

$$
\begin{aligned}
& C_{11}=\boldsymbol{p}_{s} \cdot \boldsymbol{p}_{s}=\delta^{2}+\left(\eta+\beta_{\nu} \nu_{s}+\beta_{\mu} \mu_{s}\right)^{2} \\
& C_{22}=\boldsymbol{p}_{\xi} \cdot \boldsymbol{p}_{\xi}=\beta_{\xi}^{2}=\frac{1}{\delta^{2}}, \\
& C_{12}=\boldsymbol{p}_{s} \cdot \boldsymbol{p}_{\xi}=\beta_{\xi}\left(\eta+\beta_{\nu} \nu_{s}+\beta_{\mu} \mu_{s}\right)=\frac{1}{\delta}\left(\eta+\beta_{\nu} \nu_{s}+\beta_{\mu} \mu_{s}\right) .
\end{aligned}
$$

The requirement that $C_{22}$ be finite is equivalent to the requirement that $\delta$ be positive. Since the minimum of $\delta$ as a function of $\xi$ must occur at $\xi= \pm h$, the set where $\delta>0$ is given by

$$
\nu>\sqrt{2 h|\mu|}
$$

Alternative strains. For some of our work it proves convenient to introduce the strains

$$
\delta_{ \pm}:=\delta(\nu, \mu, \pm h) \equiv \sqrt{\nu^{2} \mp 2 h \mu}
$$

in terms of which

$$
\begin{gathered}
2 \nu^{2}=\delta_{+}^{2}+\delta_{-}^{2}, \quad 4 h \mu=\delta_{-}^{2}-\delta_{+}^{2}, \quad \nu^{4}-4 h^{2} \mu^{2}=\delta_{+}^{2} \delta_{-}^{2} \\
2 \delta^{2}=\left(1+\frac{\xi}{h}\right) \delta_{+}^{2}+\left(1-\frac{\xi}{h}\right) \delta_{-}^{2}
\end{gathered}
$$

The nonconvex domain in $(\nu, \mu)$-space defined by $(2.8)$ is thus an analytic diffeomorphism of the open first quadrant in $\left(\delta_{-}, \delta_{+}\right)$-space, which in turn is an analytic diffeomorphism of the open unit disk.

The stored-energy function. We generate our constitutive equations from those of the 2-dimensional theory [2]. We assume that the 2-dimensional body is hyperelastic and homogeneous, with a stored-energy function $\Phi$ depending on (2.7). For an isotropic body, $\Phi$ depends only on $C_{11}+C_{22}$. Here we merely assume that $\Phi$ is even in $C_{12}$. Thus $\Phi$ depends only on $\delta^{2}$ and $\left(\eta+\beta_{\nu} \nu_{s}+\beta_{\mu} \mu_{s}\right)^{2}$. We define the stored-energy function $W$ for the rod by

$$
\begin{aligned}
W\left(\nu, \mu, \eta, \nu^{\prime}, \mu^{\prime}\right) & :=\int_{-h}^{h} \Phi\left(\gamma\left(\nu, \mu, \eta, \nu^{\prime}, \mu^{\prime}, \xi\right)^{2}, \delta(\nu, \mu, \xi)^{2}\right) d \xi \\
\gamma\left(\nu, \mu, \eta, \nu^{\prime}, \mu^{\prime}, \xi\right) & :=\eta+\beta_{\nu}(\nu, \mu, \xi) \nu^{\prime}+\beta_{\mu}(\nu, \mu, \xi) \mu^{\prime}
\end{aligned}
$$

with the domain of $W$ defined by (2.8). Here the primes have no operational significance: They merely identify arguments $\nu^{\prime}$ and $\mu^{\prime}$ whose slots will be occupied by $\nu_{s}$ and $\mu_{s}$. 
From (2.11) we immediately read off the symmetry condition

$$
W\left(\nu, \mu,-\eta,-\nu^{\prime},-\mu^{\prime}\right)=W\left(\nu, \mu, \eta, \nu^{\prime}, \mu^{\prime}\right)
$$

We use (2.6), (2.11), and the change of variables $\xi=-\chi$ to obtain a second symmetry condition:

$$
\begin{aligned}
& W\left(\nu,-\mu, \eta,-\nu^{\prime}, \mu^{\prime}\right) \\
& \equiv \int_{-h}^{h} \Phi\left(\left[\eta-\beta_{\nu}(\nu,-\mu, \xi) \nu^{\prime}+\beta_{\mu}(\nu,-\mu, \xi) \mu^{\prime}\right]^{2}, \delta(\nu,-\mu, \xi)^{2}\right) d \xi \\
& \equiv-\int_{h}^{-h} \Phi\left(\left[\eta+\beta_{\nu}(\nu, \mu, \chi) \nu^{\prime}+\beta_{\mu}(\nu, \mu, \chi) \mu^{\prime}\right]^{2}, \delta(\nu, \mu, \chi)^{2}\right) d \chi \\
& \equiv W\left(\nu, \mu, \eta, \nu^{\prime}, \mu^{\prime}\right) .
\end{aligned}
$$

Combining (2.13) and (2.14) we obtain a third symmetry condition:

$$
W\left(\nu,-\mu,-\eta, \nu^{\prime},-\mu^{\prime}\right)=W\left(\nu, \mu, \eta, \nu^{\prime}, \mu^{\prime}\right)
$$

These conditions imply that

$$
\begin{aligned}
W_{\nu^{\prime}}(\nu, \mu, 0,0,0) & =W_{\eta}(\nu, \mu, 0,0,0)=W_{\mu^{\prime}}(\nu, \mu, 0,0,0)=0, \\
W_{\nu^{\prime}}\left(\nu, 0, \eta, 0, \mu^{\prime}\right) & =W_{\mu}\left(\nu, 0, \eta, 0, \mu^{\prime}\right)=0, \\
W_{\eta}\left(\nu, 0,0, \nu^{\prime}, 0\right) & =W_{\mu}\left(\nu, 0,0, \nu^{\prime}, 0\right)=W_{\mu^{\prime}}\left(\nu, 0,0, \nu^{\prime}, 0\right)=0 .
\end{aligned}
$$

Dynamics. Since we are restricting our attention to homogeneous hyperelastic materials with constant mass density $\rho$, the governing partial differential equations of motion are the Euler-Lagrange equations for the Lagrangian functional $\iint_{\mathbb{R}^{2}}\left\{\int_{-h}^{h} \frac{1}{2} \rho \boldsymbol{p}_{t} \cdot \boldsymbol{p}_{t} d \xi-W\right\} d s d t$, where the term in braces, the Lagrangian, is the difference between the kinetic energy per unit length (found from $(2.5)_{3}$ ) and $W$ :

$$
\begin{gathered}
\frac{\rho}{2} \int_{-h}^{h}\left\{\left[\boldsymbol{r}_{t} \cdot \boldsymbol{a}(\theta)-\beta(\nu, \mu, \xi) \theta_{t}\right]^{2}+\left[\boldsymbol{r}_{t} \cdot \boldsymbol{b}(\theta)+\beta_{\nu}(\nu, \mu, \xi) \nu_{t}\right.\right. \\
\left.\left.+\beta_{\mu}(\nu, \mu, \xi) \mu_{t}\right]^{2}\right\} d \xi-W\left(\nu, \mu, \eta, \nu_{s}, \mu_{s}\right) .
\end{gathered}
$$

We substitute

$$
\begin{gathered}
\nu=\boldsymbol{r}_{s} \cdot \boldsymbol{a}(\theta), \quad \nu_{s}=\boldsymbol{r}_{s s} \cdot \boldsymbol{a}(\theta)+\theta_{s} \boldsymbol{r}_{s} \cdot \boldsymbol{b}(\theta), \quad \nu_{t}=\boldsymbol{r}_{s t} \cdot \boldsymbol{a}(\theta)+\theta_{t} \boldsymbol{r}_{s} \cdot \boldsymbol{b}(\theta), \\
\eta=\boldsymbol{r}_{s} \cdot \boldsymbol{b}, \quad \mu_{s}=\theta_{s s}, \quad \mu_{t}=\theta_{s t},
\end{gathered}
$$

coming from (2.4), into (2.17) to get an expression for the Lagrangian denoted by $L\left(\boldsymbol{r}_{t}, \boldsymbol{r}_{s t}, \boldsymbol{r}_{s}, \boldsymbol{r}_{s s}, \theta, \theta_{t}, \theta_{s t}, \theta_{s}, \theta_{s s}\right)$. Then the Euler-Lagrange equations are

$$
\begin{aligned}
& \frac{\partial}{\partial t} \frac{\partial L}{\partial \boldsymbol{r}_{t}}-\frac{\partial^{2}}{\partial s \partial t} \frac{\partial L}{\partial \boldsymbol{r}_{s t}}+\frac{\partial}{\partial s} \frac{\partial L}{\partial \boldsymbol{r}_{s}}-\frac{\partial^{2}}{\partial s^{2}} \frac{\partial L}{\partial \boldsymbol{r}_{s s}}=\mathbf{0}, \\
& \frac{\partial}{\partial t} \frac{\partial L}{\partial \theta_{t}}-\frac{\partial^{2}}{\partial s \partial t} \frac{\partial L}{\partial \theta_{s t}}+\frac{\partial}{\partial s} \frac{\partial L}{\partial \theta_{s}}-\frac{\partial^{2}}{\partial s^{2}} \frac{\partial L}{\partial \theta_{s s}}=\frac{\partial L}{\partial \theta} .
\end{aligned}
$$


For completeness we readily compute

$$
\begin{aligned}
\frac{\partial L}{\partial \boldsymbol{r}_{t}}= & \rho \int_{-h}^{h}\left\{\left[\boldsymbol{r}_{t} \cdot \boldsymbol{a}-\beta \theta_{t}\right] \boldsymbol{a}+\left[\boldsymbol{r}_{t} \cdot \boldsymbol{b}+\beta_{\nu} \nu_{t}+\beta_{\mu} \mu_{t}\right] \boldsymbol{b}\right\} d \xi, \\
\frac{\partial L}{\partial r_{s t}}= & \rho \int_{-h}^{h}\left[\boldsymbol{r}_{t} \cdot \boldsymbol{b}+\beta_{\nu} \nu_{t}+\beta_{\mu} \mu_{t}\right] \beta_{\nu} \boldsymbol{a} d \xi \\
\frac{\partial L}{\partial \boldsymbol{r}_{s}}= & \rho \int_{-h}^{h}\left\{-\left[\boldsymbol{r}_{t} \cdot \boldsymbol{a}-\beta \theta_{t}\right] \theta_{t} \beta_{\nu} \boldsymbol{a}+\left[\boldsymbol{r}_{t} \cdot \boldsymbol{b}+\beta_{\nu} \nu_{t}+\beta_{\mu} \mu_{t}\right]\left[\left(\beta_{\nu \nu} \nu_{t}+\beta_{\mu \nu} \mu_{t}\right) \boldsymbol{a}+\beta_{\nu} \theta_{t} \boldsymbol{b}\right]\right\} d \xi \\
& -W_{\nu} \boldsymbol{a}-\mu W_{\nu^{\prime}} \boldsymbol{b}-W_{\eta} \boldsymbol{b}, \\
\frac{\partial L}{\partial \boldsymbol{r}_{s s}}= & -W_{\nu^{\prime}} \boldsymbol{a}, \\
\frac{\partial L}{\partial \theta_{t}}= & \rho \int_{-h}^{h}\left\{-\left[\boldsymbol{r}_{t} \cdot \boldsymbol{a}-\beta \theta_{t}\right] \beta+\left[\boldsymbol{r}_{t} \cdot \boldsymbol{b}+\beta_{\nu} \nu_{t}+\beta_{\mu} \mu_{t}\right] \beta_{\nu} \eta\right\} d \xi \\
\frac{\partial L}{\partial \theta_{s t}}= & \rho \int_{-h}^{h}\left[r_{t} \cdot \boldsymbol{b}+\beta_{\nu} \nu_{t}+\beta_{\mu} \mu_{t}\right] \beta_{\mu} d \xi \\
\frac{\partial L}{\partial \theta_{s}}= & \rho \int_{-h}^{h}\left\{-\left[\boldsymbol{r}_{t} \cdot \boldsymbol{a}-\beta \theta_{t}\right] \beta_{\mu} \theta_{t}+\left[\boldsymbol{r}_{t} \cdot \boldsymbol{b}+\beta_{\nu} \nu_{t}+\beta_{\mu} \mu_{t}\right]\left[\beta_{\nu \mu} \nu_{t}+\beta_{\mu \mu} \mu_{t}\right]\right\} d \xi \\
\frac{\partial L}{\partial \theta_{s s}}= & -W_{\mu^{\prime}}, \\
\frac{\partial L}{\partial \theta}= & \rho \int_{-h}^{h}\left\{-\left[\boldsymbol{r}_{t} \cdot \boldsymbol{a}-\beta \theta_{t}\right]\left[\boldsymbol{r}_{t} \cdot \boldsymbol{b}-\beta_{\nu} \eta \theta_{t}\right]\right. \\
& \quad+\left[\boldsymbol{r}_{t} \cdot \boldsymbol{b}+\beta_{\nu} \nu_{t}+\beta_{\mu} \mu_{t}\right]\left[-\boldsymbol{r}_{t} \cdot \boldsymbol{a}+\beta_{\nu \nu} \eta \nu_{t}+\beta_{\mu \nu} \eta \mu_{t}+\beta_{\nu}\left(\boldsymbol{r}_{s t} \cdot \boldsymbol{b}-\nu \theta_{t}\right)\right\} d \xi
\end{aligned}
$$

Conserved quantities. Note that $L$ does not depend on $\boldsymbol{r}, s, t$, and $L$ is invariant under a rotation about $\boldsymbol{k}$, i.e., under transformations of the form $\boldsymbol{r} \rightarrow \boldsymbol{Q} \cdot \boldsymbol{r}, \theta \rightarrow \theta+\psi$, where $\boldsymbol{Q}$ has a matrix of the form $\left[\begin{array}{rr}\cos \psi & -\sin \psi \\ \sin \psi & \cos \psi\end{array}\right]$ with respect to the basis $\{\boldsymbol{i}, \boldsymbol{j}\}$. Noether's Theorem (cf. [9, Thm. 6.6]) ensures that the governing equations admit the standard conserved quantities: linear momentum corresponding to the absence of $\boldsymbol{r}$ as an argument of $L$, angular momentum corresponding to the invariance of $L$ under rotation about $\boldsymbol{k}$, and energy corresponding to the absence of $t$ as an argument of $L$.

For future reference, it is useful to examine the energy integral. We take the dot product of $(2.17)_{1}$ with $\boldsymbol{r}_{t}$, multiply $(2.17)_{2}$ by $\theta_{t}$, and then use identities like $\boldsymbol{r}_{t} \cdot \partial_{t}\left(\partial L / \partial \boldsymbol{r}_{t}\right)=\partial_{t}\left(\boldsymbol{r}_{t} \cdot \partial L / \partial \boldsymbol{r}_{t}\right)-\boldsymbol{r}_{t t} \cdot\left(\partial L / \partial \boldsymbol{r}_{t}\right)$ to write the sum of these products in the conservation form

$$
\frac{\partial}{\partial t}\left[\boldsymbol{r}_{t} \cdot \frac{\partial L}{\partial \boldsymbol{r}_{t}}+\boldsymbol{r}_{s t} \cdot \frac{\partial L}{\partial \boldsymbol{r}_{s t}}+\theta_{t} \frac{\partial L}{\partial \theta_{t}}+\theta_{s t} \frac{\partial L}{\partial \theta_{s t}}-L\right]+\frac{\partial F}{\partial s}=0
$$

where $F$ is a complicated expression involving $L$. We integrate (2.21) with respect to $s$ over $[a, b]$, and use (2.20) to obtain the conservation of energy:

$$
\begin{aligned}
0 & =\frac{d}{d t} \int_{a}^{b}\left\{\int_{-h}^{h} \rho\left\{\left[\boldsymbol{r}_{t} \cdot \boldsymbol{a}-\beta(\nu, \mu, \xi) \theta_{t}\right]^{2}+\left[\boldsymbol{r}_{t} \cdot \boldsymbol{b}+\beta_{\nu} \nu_{t}+\beta_{\mu} \mu_{t}\right]^{2}\right\} d \xi-L\right\} d s+\left.F\right|_{a} ^{b} \\
& =\frac{d}{d t} \int_{a}^{b}\left\{\int_{-h}^{h} \frac{\rho}{2}\left\{\left[\boldsymbol{r}_{t} \cdot \boldsymbol{a}-\beta(\nu, \mu, \xi) \theta_{t}\right]^{2}+\left[\boldsymbol{r}_{t} \cdot \boldsymbol{b}+\beta_{\nu} \nu_{t}+\beta_{\mu} \mu_{t}\right]^{2}\right\} d \xi+W\right\} d s+\left.F\right|_{a} ^{b}
\end{aligned}
$$

If the dependent variables entering the Euler-Lagrange equations (2.19) are required to have period $b-a$ in $s$, then the boundary term in (2.22) vanishes. On the other 
hand, if $\boldsymbol{r}_{t}, \boldsymbol{r}_{s t}, \theta_{t}, \theta_{s t} \rightarrow 0$ as $s \rightarrow \pm \infty$ and if appropriate derivatives of $\boldsymbol{r}$ and $\theta$ are bounded in this limit, then (2.22) holds with $a=-\infty, b=\infty$, and no "boundary" term. Of course, (2.22) is valid only when the fields are sufficiently regular; it is not valid when there are shocks. The conservation law corresponding to the absence of $s$ as an argument of $L$ can be obtained by taking the dot product of $(2.17)_{1}$ with $\boldsymbol{r}_{\boldsymbol{s}}$, multiplying $(2.17)_{2}$ by $\theta_{s}$, and proceeding as above. (This approach is easier than using $[9$, Thm. 6.6].)

Explicit integration. All the integrals in (2.20) can be evaluated in closed form, by the simple device of replacing the variable $\xi$ of integration with $\delta$ given by $(2.3)_{2}$. Indeed,

$$
\int_{-h}^{h} f(\delta, \xi) d \xi=\int_{\delta(\nu, \mu,+h)}^{\delta(\nu, \mu,-h)} f\left(\delta, \frac{\nu^{2}-\delta^{2}}{2 \mu}\right) \frac{\delta}{\mu} d \delta
$$

In particular,

$$
\begin{aligned}
\int_{-h}^{h} \beta(\nu, \mu, \xi) d \xi & =\int_{-h}^{h} \frac{\nu-\delta}{\mu} d \xi \\
& =2 h \frac{\nu}{\mu}-\frac{1}{\mu^{2}} \int_{\delta(\nu, \mu,+h)}^{\delta(\nu, \mu,-h)} \delta^{2} d \delta \\
& =\frac{1}{\mu^{2}}\left[2 h \nu \mu-\frac{1}{3} \delta(\nu, \mu,-h)^{3}+\frac{1}{3} \delta(\nu, \mu, h)^{3}\right]
\end{aligned}
$$

L'Hôpital's Rule ensures that the limit of this expression at $\mu=0$ is 0 . Indeed, the derivative of the numerator of the last form of (2.24) with respect to $\mu$ is $h[2 \nu-$ $\delta(\nu, \mu,-h)-\delta(\nu, \mu, h)]$, and the derivative of this expression with respect to $\mu$ is $h^{2}\left[-\delta(\nu, \mu,-h)^{-1}+\delta(\nu, \mu, h)^{-1}\right]$, whose limit at $\mu=0$ is 0 . The same kind of argument handles other cases in which $\mu$ appears in the denominator.

3. Equations of travelling waves. We seek solutions of the equations of motion in which each dependent variable $f$ has the form $f(s, t)=\hat{f}(s-c t)$ where $c$ is a constant. We denote the phase $s-c t$ by $\sigma$, henceforth denote derivatives of these variables with respect to $\sigma$ by a prime, and drop the circumflex. We set

$$
\Psi(\delta, \gamma):=\Phi\left(\delta^{2}, \gamma^{2}\right)
$$

In view of (2.11), the formula (2.17) for the Lagrangian reduces to

$$
\begin{array}{r}
\Lambda\left(\nu, \mu, \eta, \nu^{\prime}, \mu^{\prime}\right):=\int_{-h}^{h}\left\{\frac{1}{2} \rho c^{2}\left[\gamma\left(\nu, \mu, \eta, \nu^{\prime}, \mu^{\prime}, \xi\right)^{2}+\delta(\nu, \mu, \xi)^{2}\right]\right. \\
\left.-\Psi\left(\gamma\left(\nu, \mu, \eta, \nu^{\prime}, \mu^{\prime}, \xi\right), \delta(\nu, \mu, \xi)\right)\right\} d \xi
\end{array}
$$

Note that this Lagrangian functional, which is the difference between the kinetic and potential energy for the rod, does not have the form of the Lagrangian functional for the dynamics of systems of rigid bodies because it need not be quadratic in the derivatives and because the potential-energy term depends on derivatives. 
The Euler-Lagrange equations for (3.2) reduce to

$0=\Lambda_{\eta} \equiv \int_{-h}^{h}\left(\rho c^{2} \gamma-\Psi_{\gamma}\right) d \xi$

$0=\frac{d \Lambda_{\nu^{\prime}}}{d \sigma}-\Lambda_{\nu} \equiv \frac{d}{d \sigma} \int_{-h}^{h}\left(\rho c^{2} \gamma-\Psi_{\gamma}\right) \beta_{\nu} d \xi-\int_{-h}^{h}\left\{\left[\rho c^{2} \gamma-\Psi_{\gamma}\right] \gamma_{\nu}+\left[\rho c^{2} \delta-\Psi_{\delta}\right] \delta_{\nu}\right\} d \xi$

$0=\frac{d \Lambda_{\mu^{\prime}}}{d \sigma}-\Lambda_{\mu} \equiv \frac{d}{d \sigma} \int_{-h}^{h}\left(\rho c^{2} \gamma-\Psi_{\gamma}\right) \beta_{\mu} d \xi-\int_{-h}^{h}\left\{\left[\rho c^{2} \gamma-\Psi_{\gamma}\right] \gamma_{\mu}+\left[\rho c^{2} \delta-\Psi_{\delta}\right] \delta_{\mu}\right\} d \xi$

where $\gamma_{\nu}=\beta_{\nu \nu} \nu^{\prime}+\beta_{\nu \mu} \mu^{\prime}$, etc. As is clear from (3.2) and (3.3a), the role of the shear strain and its corresponding resultant is degenerate.

Since $\Lambda$ does not depend on $\sigma$, the Euler-Lagrange equations for $\Lambda$ have a corresponding energy-like integral. We obtain it by multiplying the $\sigma$-derivative of (3.3a) by $\eta$, multiplying (3.3b) by $\nu^{\prime}$, multiplying (3.3c) by $\mu^{\prime}$, and adding these products to obtain

$$
\eta \Lambda_{\eta}+\nu^{\prime} \Lambda_{\nu^{\prime}}+\mu^{\prime} \Lambda_{\mu^{\prime}}-\Lambda \equiv \int_{-h}^{h}\left\{\frac{1}{2} \rho c^{2}\left(\gamma^{2}-\delta^{2}\right)+\Psi-\gamma \Psi_{\gamma}\right\} d \xi=\text { const. }
$$

Note that this term is not the integral with respect to $\xi$ over $[-h, h]$ of the kinetic and potential energy densities, which is $\int_{-h}^{h}\left\{\frac{1}{2} \rho c^{2}\left(\gamma^{2}+\delta^{2}\right)+\Psi\right\} d \xi$.

It is instructive to examine the true conservation of energy $(2.22)$ in the context of periodic travelling waves. We first note that if $\hat{u}$ has period $b-a$ and if $u(s, t)=$ $\hat{u}(s-c t)$, then $u(\cdot, t)$ has period $b-a$. We replace all the functions of $(s, t)$ in the second integrand of (2.22) by functions of $s-c t$ of period $b-a$, and make the change of variable $\sigma=s-c t$ to obtain

$$
\begin{aligned}
0 & =\frac{d}{d t} \int_{a}^{b} \int_{-h}^{h}\left\{\frac{1}{2} \rho c^{2}\left(\gamma^{2}+\delta^{2}\right)+\Psi\right\} d \xi d s=\frac{d}{d t} \int_{a-c t}^{b-c t} \int_{-h}^{h}\left\{\frac{1}{2} \rho c^{2}\left(\gamma^{2}+\delta^{2}\right)+\Psi\right\} d \xi d \sigma \\
& =-\left.c \int_{-h}^{h}\left\{\frac{1}{2} \rho c^{2}\left(\gamma^{2}+\delta^{2}\right)+\Psi\right\} d \xi\right|_{\sigma=a-c t} ^{\sigma=b-c t}
\end{aligned}
$$

For travelling waves with period $b-a$, this is an identity. The same observation holds for other such conservation laws.

It proves convenient to introduce a Hamiltonian $\Lambda^{*}$ when possible. For this purpose we set

$$
\begin{aligned}
H: & =\Lambda_{\eta}\left(\nu, \mu, \eta, \nu^{\prime}, \mu^{\prime}\right) \\
& =\int_{-h}^{h}\left\{\rho c^{2} \gamma\left(\nu, \mu, \eta, \nu^{\prime}, \mu^{\prime}, \xi\right)-\Psi_{\gamma}\left(\gamma\left(\nu, \mu, \eta, \nu^{\prime}, \mu^{\prime}, \xi\right), \delta(\nu, \mu, \xi)\right)\right\} d \xi \\
\bar{N}: & =\Lambda_{\nu^{\prime}}\left(\nu, \mu, \eta, \nu^{\prime}, \mu^{\prime}\right) \\
& =\int_{-h}^{h}\left\{\rho c^{2} \gamma\left(\nu, \mu, \eta, \nu^{\prime}, \mu^{\prime}, \xi\right)-\Psi_{\gamma}\left(\gamma\left(\nu, \mu, \eta, \nu^{\prime}, \mu^{\prime}, \xi\right), \delta(\nu, \mu, \xi)\right)\right\} \beta_{\nu}(\nu, \mu, \xi) d \xi \\
\bar{M}: & =\Lambda_{\mu^{\prime}}\left(\nu, \mu, \eta, \nu^{\prime}, \mu^{\prime}\right) \\
& =\int_{-h}^{h}\left\{\rho c^{2} \gamma\left(\nu, \mu, \eta, \nu^{\prime}, \mu^{\prime}, \xi\right)-\Psi_{\gamma}\left(\gamma\left(\nu, \mu, \eta, \nu^{\prime}, \mu^{\prime}, \xi\right), \delta(\nu, \mu, \xi)\right)\right\} \beta_{\mu}(\nu, \mu, \xi) d \xi .
\end{aligned}
$$


We regard this as a system to be solved for $\eta, \nu^{\prime}, \mu^{\prime}$ as functions of $\nu, \mu, H, \bar{N}, \bar{M}$. The quadratic form of the second derivatives of $\Lambda$ with respect to $\eta, \nu^{\prime}, \mu^{\prime}$ is

$$
\int_{-h}^{h}\left[\rho c^{2}-\Psi_{\gamma \gamma}\right]\left[a+b \beta_{\nu}+c \beta_{\mu}\right]^{2} d \xi, \quad a, b, c \in \mathbb{R} .
$$

If we assume that $\rho c^{2}-\Psi_{\gamma \gamma}$ is everywhere positive or everywhere negative, then (3.7) is positive- or negative-definite (because the form of $\beta$ prevents $a+b \beta_{\nu}+c \beta_{\mu}$ from vanishing for all $\xi \in(-h, h)$ ). Thus $\Lambda$ is respectively convex or concave in $\eta, \nu^{\prime}, \mu^{\prime}$. If we strengthen the assumption that $\rho c^{2}-\Psi_{\gamma \gamma}$ have fixed sign by requiring that $\Lambda \rightarrow \pm \infty$ as $|\eta|+\left|\nu^{\prime}\right|+\left|\mu^{\prime}\right| \rightarrow \infty$, i.e., by requiring that $\Psi$ be asymptotic strictly sub- or superquadratic in $\eta, \nu^{\prime}, \mu^{\prime}$, then (3.6) can be uniquely solved for $\eta, \nu^{\prime}, \mu^{\prime}$ as functions of the other variables in these equations. (The proof consists in showing that these conditions imply that $\left(\eta, \nu^{\prime}, \mu^{\prime}\right) \mapsto \Lambda\left(\nu, \mu, \eta, \nu^{\prime}, \mu^{\prime}\right)-H \eta-\bar{N} \nu^{\prime}-\bar{M} \mu^{\prime}$ has a unique minimum or maximum.) We denote these solutions by

$$
\eta=\eta^{\sharp}(\nu, \mu, H, \bar{N}, \bar{M}), \quad \nu^{\prime}=\nu^{\sharp}(\nu, \mu, H, \bar{N}, \bar{M}), \quad \mu^{\prime}=\mu^{\sharp}(\nu, \mu, H, \bar{N}, \bar{M}) .
$$

We now define the Hamiltonian $\Lambda^{*}$ by the Legendre transform (cf. (3.4))

$$
\begin{aligned}
\Lambda^{*}(\nu, \mu, H, \bar{N}, \bar{M}):= & \eta^{\sharp}(\nu, \mu, H, \bar{N}, \bar{M}) H+\nu^{\sharp}(\nu, \mu, H, \bar{N}, \bar{M}) \bar{N}+\mu^{\sharp}(\nu, \mu, H, \bar{N}, \bar{M}) \bar{M} \\
& -\Lambda\left(\nu, \mu, \eta^{\sharp}(\nu, \mu, H, \bar{N}, \bar{M}), \nu^{\sharp}(\nu, \mu, H, \bar{N}, \bar{M}), \mu^{\sharp}(\nu, \mu, H, \bar{N}, \bar{M})\right) \\
\equiv & \int_{-h}^{h}\left\{\frac{1}{2} \rho c^{2}\left(\gamma^{2}-\delta^{2}\right)+\Psi-\gamma \Psi_{\gamma}\right\} d \xi
\end{aligned}
$$

where the arguments of $\gamma \quad$ are $\left(\nu, \mu, \eta^{\sharp}(\nu, \mu, H, \bar{N}, \bar{M}), \quad \nu^{\sharp}(\nu, \mu, H, \bar{N}, \bar{M})\right.$, $\left.\mu^{\sharp}(\nu, \mu, H, \bar{N}, \bar{M}), \xi\right)$, the arguments of $\delta$ are $(\nu, \mu, \xi)$, and the arguments of $\Psi$ are $\left(\nu, \mu, \eta^{\sharp}(\nu, \mu, H, \bar{N}, \bar{M}), \quad \nu^{\sharp}(\nu, \mu, H, \bar{N}, \bar{M}), \quad \mu^{\sharp}(\nu, \mu, H, \bar{N}, \bar{M})\right)$. Equation (3.4) ensures that $\Lambda^{*}$ is conserved. Note that

$$
\Lambda_{\nu}^{*}=-\Lambda_{\nu}, \quad \Lambda_{\mu}^{*}=-\Lambda_{\mu}, \quad \Lambda_{H}^{*}=\eta^{\sharp}, \quad \Lambda_{\bar{N}}^{*}=\nu^{\sharp}, \quad \Lambda_{\bar{M}}^{*}=\mu^{\sharp}
$$

where the arguments of $\Lambda^{*}$ and its derivatives are $\nu, \mu, H, \bar{N}, \bar{M}$, the arguments of $\Lambda$ and its derivatives are $\nu, \mu, \eta^{\sharp}, \nu^{\sharp}, \mu^{\sharp}$, and the arguments of $\eta^{\sharp}, \nu^{\sharp}, \mu^{\sharp}$ are $\nu, \mu, H, \bar{N}, \bar{M}$.

When $\rho c^{2}>\Psi_{\gamma \gamma}$ everywhere, the speed of propagation exceeds the natural speed of what might be termed shear waves, in which case the speed $c$ of propagation for such waves can be termed supersonic in shear. When the opposite inequality holds, $c$ can be termed subsonic in shear. Likewise, when $\rho c^{2}>\Psi_{\delta \delta}$, the speed of propagation exceeds the natural speed of what might be termed extensional-flexural waves, in which case $c$ is supersonic for extensional-flexural motions.

Equations (3.6) and (3.10) imply that the Euler-Lagrange equations (3.3) are equivalent to Hamilton's equations

$$
\frac{d}{d \sigma} \nu=\Lambda_{\bar{N}}^{*}, \quad \frac{d}{d \sigma} \mu=\Lambda_{\bar{M}}^{*}, \quad H=0, \quad \frac{d}{d \sigma} \bar{N}=-\Lambda_{\nu}^{*}, \quad \frac{d}{d \sigma} \bar{M}=-\Lambda_{\mu}^{*} .
$$

By construction, $\Lambda^{*}$ is convex or concave in $H, \bar{N}, \bar{M}$ according as $\Lambda$ is convex or concave in $\eta, \nu^{\prime}, \mu^{\prime}$. But even in this special case predicated on the invertibility of (3.6), the Hamiltonian $\Lambda^{*}$ appears to lack some attractive mathematical structure. E.g., even in the simple examples we shall consider, it does not appear that level surfaces of $\Lambda^{*}$ enclose convex regions or even regions star-shaped with respect to an 
interior point. (In particular, if $\Psi$ blows up to infinity at a sufficiently fast rate as $\delta \searrow 0$, then $W$ would blow up on the non-convex boundary of its domain of definition, defined by (2.8), an impossibility for convex functions.) This observation means that many powerful tools for the qualitative treatment of Hamiltonian equations, like those described in $[6,10]$, are not available to us. See Section 5. We do note, however, that the positivity of $\nu^{\sharp}$ ensures that the gradient of $\Lambda^{*}$ vanishes nowhere, so that the differentiability of $\Lambda^{*}$ ensures that surfaces $\Lambda^{*}(\nu, \mu, H, \bar{N}, \bar{M})=E$ of constant energy are of class $C^{1}$.

Since (3.11) implies that $H=0$, we can make this substitution in (3.9). In place of level surfaces of $\Lambda^{*}$, we restrict our attention to the intersections of such surfaces with the plane $H=0: \Lambda^{*}(\nu, \mu, 0, \bar{N}, \bar{M})=E$.

4. Example. Let

$$
2 \Psi(\gamma, \delta)=A \gamma^{2}+B \delta^{2}+\varphi(\delta)
$$

where $A$ and $B$ are positive with $A \equiv \Psi_{\gamma \gamma} \neq \rho c^{2}$ and where $\varphi$ is positive-valued with $\varphi(\delta) \rightarrow \infty$ as $\delta \rightarrow 0$. Then $\Lambda$ has the form

$\Lambda\left(\nu, \mu, \eta, \nu^{\prime}, \mu^{\prime}\right)=\frac{1}{2} \int_{-h}^{h}\left\{\left(\rho c^{2}-A\right) \gamma\left(\nu, \mu, \eta, \nu^{\prime}, \mu^{\prime}, \xi\right)^{2}+\left(\rho c^{2}-B\right) \delta(\nu, \mu, \xi)^{2}-\varphi(\delta(\nu, \mu, \xi))\right\} d \xi$.

In this case, (3.6) reduces to

$$
H:=\left(\rho c^{2}-A\right) \int_{-h}^{h} \gamma d \xi, \quad \bar{N}=\left(\rho c^{2}-A\right) \int_{-h}^{h} \gamma \beta_{\nu} d \xi, \quad \bar{M}=\left(\rho c^{2}-A\right) \int_{-h}^{h} \gamma \beta_{\mu} d \xi
$$

Let us set

$$
\begin{aligned}
& \mathbf{g}_{0}:=\left(\int 1, \int \beta_{\nu}, \int \beta_{\mu}\right)^{\top}, \quad \mathbf{g}_{1}:=\left(\int \beta_{\nu}, \int \beta_{\nu}^{2}, \int \beta_{\nu} \beta_{\mu}\right)^{\top} \\
& \mathbf{g}_{2}:=\left(\int \beta_{\mu}, \int \beta_{\nu} \beta_{\mu}, \int \beta_{\mu}^{2}\right)^{\top}, \quad \boldsymbol{n}:=(H, \bar{N}, \bar{M})^{\top}
\end{aligned}
$$

with the integrals taken over $(-h, h)$ and with $\mathrm{T}$ denoting the transpose. Then Cramer's Rule implies that

$$
\begin{aligned}
\eta^{\sharp}=\frac{\operatorname{det}\left(\mathbf{n}, \mathbf{g}_{1}, \mathbf{g}_{2}\right)}{\left(\rho c^{2}-A\right) \Delta}, \quad \nu^{\sharp} & =\frac{\operatorname{det}\left(\mathbf{g}_{0}, \mathbf{n}, \mathbf{g}_{2}\right)}{\left(\rho c^{2}-A\right) \Delta}, \quad \mu^{\sharp}=\frac{\operatorname{det}\left(\mathbf{g}_{0}, \mathbf{g}_{1}, \mathbf{n}\right)}{\left(\rho c^{2}-A\right) \Delta}, \\
\Delta: & =\operatorname{det}\left(\mathbf{g}_{0}, \mathbf{g}_{1}, \mathbf{g}_{2}\right) .
\end{aligned}
$$

Now $\Lambda^{*}$ has the value

$$
\eta \Lambda_{\eta}+\nu^{\prime} \Lambda_{\nu^{\prime}}+\mu^{\prime} \Lambda_{\mu^{\prime}}-\Lambda=\frac{1}{2} \int_{-h}^{h}\left[\left(\rho c^{2}-A\right) \gamma^{2}-\left(\rho c^{2}-B\right) \delta^{2}+\varphi(\delta)\right] d \xi
$$

so that

$$
\begin{aligned}
\Lambda^{*}(\nu, \mu, H, \bar{N}, \bar{M})=\frac{1}{2} \int_{-h}^{h}\{ & \left(\rho c^{2}-A\right) \gamma\left(\nu, \mu, \eta^{\sharp}, \nu^{\sharp}, \mu^{\sharp}, \xi\right)^{2} \\
& \left.-\left(\rho c^{2}-B\right) \delta(\nu, \mu, \xi)^{2}+\varphi(\delta(\nu, \mu, \xi))\right\} d \xi .
\end{aligned}
$$


The substitution of (4.5) into the first term of (4.7) yields

$$
\frac{\rho c^{2}-A}{2} \int_{-h}^{h} \gamma^{2} d \xi=\frac{1}{2\left(\rho c^{2}-A\right) \Delta^{2}} \int_{-h}^{h}\left[\omega_{1} H+\omega_{2} \bar{N}+\omega_{3} \bar{M}\right]^{2} d \xi
$$

where

$$
\begin{aligned}
\omega_{1}=\operatorname{det}\left(\mathbf{g}, \mathbf{g}_{1}, \mathbf{g}_{2}\right), & \omega_{2}=\operatorname{det}\left(\mathbf{g}_{0}, \mathbf{g}, \mathbf{g}_{2}\right), \quad \omega_{3}=\operatorname{det}\left(\mathbf{g}_{0}, \mathbf{g}_{1}, \mathbf{g}\right), \\
& \mathbf{g}:=\left(1, \beta_{\nu}, \beta_{\mu}\right) .
\end{aligned}
$$

Expression (4.8) is a definite quadratic form in $H, \bar{N}, \bar{M}$ with coefficients depending on $\nu, \mu$. In view of (2.23), we also have

$$
\int_{-h}^{h}\left\{\left(\rho c^{2}-B\right) \delta(\nu, \mu, \xi)^{2}-\varphi(\delta(\nu, \mu, \xi))\right\} d \xi=2 h\left(\rho c^{2}-B\right) \nu^{2}-\frac{1}{\mu} \int_{\delta_{+}}^{\delta_{-}} \varphi(\delta) \delta d \delta .
$$

In particular, for $\varphi(\delta)=\delta^{-p}$ with $p \neq 2$,

$$
\int_{-h}^{h} \delta^{-r} d \xi=\frac{\delta_{-}^{-r+2}-\delta_{+}^{-r+2}}{(2-r) \mu}=\frac{4 h}{2-r} \frac{\delta_{-}^{-r+2}-\delta_{+}^{-r+2}}{\delta_{-}^{2}-\delta_{+}^{2}}
$$

with the special values

$$
\int_{-h}^{h} \delta^{-1} d \xi=\frac{4 h}{\delta_{+}+\delta_{-}}, \quad \int_{-h}^{h} \delta^{-4} d \xi=\frac{2 h}{\nu^{4}-4 h^{2} \mu^{2}}=\frac{2 h}{\delta_{+}^{2} \delta_{-}^{2}}
$$

Each of these functions of $\delta_{ \pm}$is convex, but only the latter has the desirable property that it blows up as $\delta_{-} \rightarrow 0$ or $\delta_{+} \rightarrow 0$.

A level surface of $\Lambda^{*}$ has the form

$$
\begin{aligned}
2 \Lambda^{*}(\nu, \mu, H, \bar{N}, \bar{M}) \equiv & \frac{1}{\left(\rho c^{2}-A\right) \Delta^{2}} \int_{-h}^{h}\left[\omega_{1} H+\omega_{2} \bar{N}+\omega_{3} \bar{M}\right]^{2} d \xi-2 h\left(\rho c^{2}-B\right) \nu^{2} \\
& +\frac{1}{\mu} \int_{\delta_{+}}^{\delta_{-}} \varphi(\delta) \delta d \delta=2 E .
\end{aligned}
$$

It does not appear that this surface or even its projection onto the plane $H=0$ is star-shaped with respect to any point within it. (Such a property plays an important role in the much of the theory of Hamiltonian differential equations.)

5. Periodic travelling waves. During the past twenty-five years, there has been an extensive development of the theory of Hamiltonian ordinary differential equations (see [14] and the references cited therein). In this section, we pluck some of the most accessible fruits of this labor and apply them to some representative problems of travelling waves. In the Conclusion, we comment briefly on the general problem. Throughout this section, we take $H=0$, in keeping with $(3.11)_{3}$.

We begin with one of the earliest results on Hamiltonian systems, due to Seifert [15] (cf. [13]):

5.1. TheOREm. Let $\mathcal{D}$ be a simply-connected domain of $\mathbb{R}^{2 n}$, let $\mathcal{D} \ni(\mathbf{q}, \mathbf{p}) \mapsto$ $\mathbf{H}(\mathbf{q}, \mathbf{p})=\mathbf{p} \cdot \mathbf{A}(\mathbf{q}) \cdot \mathbf{p}+\mathrm{V}(\mathbf{q}) \in \mathcal{R}$ be continuously differentiable (here $\mathbf{A}$ is an $n \times n$ matrix-valued function and $\mathbf{p} \cdot \mathbf{A}(\mathbf{q}) \cdot \mathbf{p}$ is its quadratic form in $\mathbf{p})$, for $\mathrm{V}_{0} \in \mathbb{R}$ let $\mathcal{V}:=\left\{\mathbf{q} \in \mathcal{D}: \mathrm{V}(\mathbf{q}) \leq \bigvee_{0}\right\}$ be diffeomorphic to the closed unit ball in $\mathbb{R}^{n}$, and let $\mathbf{A}(\mathbf{q})$ 
be uniformly positive-definite for $\mathbf{q} \in \mathcal{V}$. Then there is a $T>0$ and a pair of points $\mathbf{q}_{0}, \mathbf{q}_{1}$ on $\partial \mathcal{V}$ such that Hamilton's equations

$$
\frac{d \mathbf{q}}{d t}=\mathrm{H}_{\mathbf{p}}(\mathbf{q}, \mathbf{p}), \quad \frac{d \mathbf{p}}{d t}=-\mathrm{H}_{\mathbf{q}}(\mathbf{q}, \mathbf{p})
$$

have a solution of period $2 T$ satisfying $(\mathbf{q}(0), \mathbf{p}(0))=\left(\mathbf{q}_{0}, \mathbf{o}\right),(\mathbf{q}(T), \mathbf{p}(T))=\left(\mathbf{q}_{1}, \mathbf{o}\right)$ with $\mathbf{q}(t)$ lying in the interior of $\mathcal{V}$ for all $t$ in $(0, T)$.

5.3. Theorem. Let $\Lambda$ be given by (4.2) with $\varphi(\delta)=C \delta^{-4}, C>0$, so that $\Lambda^{*}$ is given by the appropriate versions of (4.7) and (4.13). Let $B>\rho c^{2}>A$. Then Hamilton's equations (3.11), which are equivalent to the Euler-Lagrange equations (3.3) for (3.2), have a nontrivial periodic solution.

Proof. We identify q with $(\nu, \mu)$, $\mathbf{p}$ with $(\bar{N}, \bar{M}), \mathbf{H}$ with $\Lambda^{*}, \mathbf{p} \cdot \mathbf{A}(\mathbf{q}) \cdot \mathbf{p}$ with (4.8), and $2 \mathrm{~V}$ with $h\left(B-\rho c^{2}\right) \nu^{2}+\frac{h C}{\nu^{4}-4 h^{2} \mu^{2}}$. We use the diffeomorphisms (2.9) and (2.10) to write $2 \mathrm{~V}=h\left(B-\rho c^{2}\right)\left(\delta_{+}^{2}+\delta_{-}^{2}\right)+\frac{h C}{\delta_{+}^{2} \delta_{-}^{2}}$. A computation of the Hessian of this function shows that it is convex on its domain, which is the first open quadrant in $\left(\delta_{+}, \delta_{+}\right)$-space. In view of the smoothness of the set $\partial \mathcal{V}$ and of the growth of $\boldsymbol{V}$ at the extremes of its domain, the (convex) set where this $\mathrm{V} \leq \mathrm{V}_{0}$ is diffeomorphic to the closed unit ball in $\mathbb{R}^{n}$. $\mathrm{u}$

It is tempting to treat the problem with the reversed inequality $B<\rho c^{2}<A$ on the wave speed by identifying $\mathrm{H}$ with $-\Lambda^{*}$, but doing so is thwarted by the physical requirement that $\int_{-h}^{h} \varphi(\delta) d \xi \rightarrow \infty$ as $\delta_{ \pm} \rightarrow 0$.

The use of Theorem 5.1 for our problems rests upon the quadratic dependence of $\Lambda^{*}$ on $\bar{N}, \bar{M}$, which requires that $\Psi$ be quadratic in $\gamma$. The limitation of $\Psi$ to such a form would be unduly restrictive for our problems of elasticity. As we mentioned earlier, many other far more refined results for Hamiltonian systems likewise impose restrictions that are severe in the context of elasticity. A powerful generalization of the theorem of Seifert, due to Hofer and Zehnder [7], and refined by Rabinowitz [12] (cf. [13]), will allow a more general material response:

5.4. Theorem. Let $\mathrm{H}$ be continuously differentiable and let $\mathrm{H}^{-1}(\mathrm{E}):=\{(\mathbf{q}, \mathbf{p})$ : $\mathrm{H}(\mathbf{q}, \mathbf{p})=\mathrm{E}\}$ be a compact hypersurface in $\mathbb{R}^{2 n}$ (on which $\left(\mathrm{H}_{\mathbf{q}}(\mathbf{q}, \mathbf{p}), \mathrm{H}_{\mathbf{p}}(\mathbf{q}, \mathbf{p})\right.$ ) vanishes nowhere). Then either (i) there are uncountably many $\varepsilon$ near 0 such that $\mathrm{H}^{-1}(\mathrm{E}+\varepsilon)$ contains a periodic solution of Hamilton's equations (5.2), or (ii) there is an infinite sequence $\varepsilon_{k} \rightarrow 0$ such that $\mathrm{H}^{-1}\left(\mathrm{E}+\varepsilon_{k}\right)$ contains uncountably many distinct periodic solutions of Hamilton's equations.

To exploit this result by identifying $\mathrm{H}$ with $\Lambda^{*}$ we assume (for simplicity of exposition) that

$$
\Psi(\gamma, \delta)=G(\gamma)+D(\delta)
$$

so that

$$
\Lambda^{*}=\int_{-h}^{h}\left[\frac{1}{2} \rho c^{2} \gamma^{2}+G(\gamma)-\gamma G_{\gamma}(\gamma)+D(\delta)-\frac{1}{2} \rho c^{2} \delta^{2}\right] d \xi
$$


We require $G$ and $D$ to satisfy the constitutive restrictions

$$
\int_{-h}^{h}\left[D(\delta)-\frac{1}{2} \rho c^{2} \delta^{2}\right] d \xi \equiv \frac{4 h}{\delta_{-}^{2}-\delta_{+}^{2}} \int_{\delta_{+}}^{\delta_{-}}\left[D(\delta)-\frac{1}{2} \rho c^{2} \delta^{2}\right] \delta d \delta \rightarrow \infty \quad \text { as } \quad\left\{\begin{array}{l}
\delta_{-} \rightarrow 0 \\
\delta_{+} \rightarrow 0 \\
\delta_{-} \rightarrow \infty \\
\delta_{+} \rightarrow \infty
\end{array}\right.
$$

An example of a function $G$ satisfying these restrictions has the form $G(\gamma)=C|\gamma|^{p}$, $0 \leq p<2$, for $|\gamma|$ sufficiently large and is suitably adjusted for $|\gamma|$ small. A function $D$ with these properties is given by $D(\delta)=C_{1} \delta^{q}+C_{2} \delta^{-r}$ with $q>2$ and $r>2$ (see (4.11)). (Theorem 5.3 handles a case in which $p=2=q$.)

Under these conditions we want to determine how $\Lambda^{*}$ depends on $\bar{N}$ and $\bar{M}$. Equation (3.6) implies that

$$
\bar{N}_{\nu^{\prime}}:=\Lambda_{\nu^{\prime} \nu^{\prime}}=\int_{-h}^{h}\left[\rho c^{2}-G_{\gamma \gamma}\right] \beta_{\nu}^{2} d \xi,
$$

so that (2.3), (2.6), (5.7) imply that

$$
\begin{gathered}
\bar{N}_{\nu^{\prime}} \geq C \int_{-h}^{h} \beta_{\nu}^{2} d \xi \equiv C \int_{-h}^{h} \frac{\beta^{2}}{\delta^{2}} d \xi \geq C \int_{-h}^{h} \frac{\xi^{2}}{\left[\max \left\{\delta_{-}, \delta_{+}\right\}\right]^{4}} d \xi=\frac{C}{\left[\max \left\{\delta_{-}, \delta_{+}\right\}\right]^{4}}, \\
\bar{N}_{\nu^{\prime}} \leq \rho c^{2} \int_{-h}^{h} \beta_{\nu}^{2} d \xi \leq \frac{C}{\left[\min \left\{\delta_{-}, \delta_{+}\right\}\right]^{4}} .
\end{gathered}
$$

Thus for fixed values of $\nu, \mu, \eta, \mu^{\prime}$, the function $\bar{N} \rightarrow \pm \infty$ if and only if $\nu^{\prime} \rightarrow \pm \infty$. Likewise, for fixed values of $\nu, \mu, \eta, \nu^{\prime}$, the function $\bar{M} \rightarrow \pm \infty$ if and only if $\mu^{\prime} \rightarrow \pm \infty$. These considerations show that a level surface of $\Lambda^{*}$ is a compact hypersurface in the open subset of $(\nu, \mu, \bar{N}, \bar{M})$-space satisfying (2.8). Recall that at the end of Section 3 we showed that the gradient of $\Lambda^{*}$ could vanish nowhere. Hence Theorem 5.4 implies

5.13. Theorem. Let (5.5)-(5.9) hold. Let $\mathrm{H}$ be identified with $\Lambda^{*}$. Then the conclusion of Theorem 5.4 holds.

Another generalization of Theorem 5.1, due to Rabinowitz [11, Thm. 1.4], which lacks the richness of the multiplicity results of Theorem 5.4 for level surfaces near a given one but guarantees a periodic solution on a given level surface, will allow us to account for other constitutive restrictions:

5.14. Theorem. Let $\mathrm{H}(\mathbf{q}, \mathbf{p})=\mathrm{K}(\mathbf{q}, \mathbf{p})+\mathrm{V}(\mathbf{q})$ where $\mathrm{K} \in C^{2}\left(\mathcal{Q} \times \mathbb{R}^{n}, \mathbb{R}\right)$, $\mathrm{V} \in C^{1}(\mathcal{Q}, \mathbb{R})$, and $\mathcal{Q}$ is a simply-connected domain in $\mathbb{R}^{n}$. Let $\mathrm{E}$ be a given number. Let

(i) $\mathrm{V}_{\mathbf{q}} \neq \mathbf{o}$ for $\mathbf{q} \in \partial \mathcal{V}(\mathrm{E})$ where $\mathcal{V}(\mathrm{E}):=\{\mathbf{q} \in \mathcal{Q}: \mathrm{V}(\mathbf{q})<\mathrm{E}\}$.

(ii) $\mathcal{V}$ is $C^{2}$-diffeomorphic to the open unit ball in $\mathbb{R}^{n}$.

(iii) For $\mathbf{q}$ in the closure of $\mathcal{V}(\mathrm{E})$,

$$
\mathrm{K}(\mathbf{q}, \mathbf{o})=0, \quad \mathbf{p} \cdot \mathrm{K}_{\mathbf{p}}(\mathbf{q}, \mathbf{p})>0 \quad \text { for } \quad \mathbf{p} \neq \mathbf{o} .
$$


(iv) For fixed $\mathbf{q} \in \mathcal{V}$ and for $|\mathbf{p}|=1$,

$$
\lim _{\alpha \rightarrow \infty} \mathrm{K}(\mathbf{q}, \alpha \mathbf{p})>\mathrm{E}-\mathrm{V}(\mathbf{q}) .
$$

Then Hamilton's equations have a (nonconstant) periodic solution on $\mathrm{H}^{-1}(\mathrm{E})$.

This Theorem, while it is couched in terms of the Hamiltonian, emphasizes the split between the $\mathrm{K}$ and $\mathrm{V}$, and is thus closer to the physical basis of our travellingwave equations than is Theorem 5.4.

To exploit this theorem, we assume that $\rho c^{2}-\Psi_{\gamma \gamma}$ is everywhere positive and that (5.5) holds, and we identify

$$
\begin{gathered}
\mathbf{q}=(\nu, \mu), \quad \mathbf{p}=(\bar{N}, \bar{M}), \quad \mathbf{H}=\Lambda^{*}, \quad \mathcal{Q}=\{(\nu, \mu):(2.8) \text { holds }\} \\
\mathbf{K}=\int_{-h}^{h}\left[\frac{1}{2} \rho c^{2} \gamma^{2}+G(\gamma)-\gamma G_{\gamma}(\gamma)\right] d \xi \\
\quad \mathrm{V}=\int_{-h}^{h}\left[D(\delta)-\frac{1}{2} \rho c^{2} \delta^{2}\right] d \xi
\end{gathered}
$$

(see (3.9)). Let us now study the hypotheses (i)-(iv).

With a slight abuse of notation, (2.6) and (2.23) imply that

$$
\begin{aligned}
\mathrm{V}_{\nu} & =\int_{-h}^{h}\left[D_{\delta}(\delta)-\rho c^{2} \delta\right] \delta_{\nu} d \xi=\nu \int_{-h}^{h}\left[\delta^{-1} D_{\delta}(\delta)-\rho c^{2}\right] d \xi \\
& =\frac{\nu}{\mu} \int_{\delta_{+}}^{\delta_{-}} D_{\delta}(\delta) d \delta-2 h \rho c^{2} \nu=\frac{\nu}{\mu}\left[D\left(\delta_{-}\right)-D\left(\delta_{+}\right)\right]-2 h \rho c^{2} \nu, \\
\mathrm{V}_{\mu} & =\int_{-h}^{h}\left[D_{\delta}(\delta)-\rho c^{2} \delta\right] \delta_{\mu} d \xi=-\int_{-h}^{h}\left[\delta^{-1} D_{\delta}(\delta)-\rho c^{2}\right] \xi d \xi \\
& =-\frac{1}{2 \mu^{2}} \int_{\delta_{+}}^{\delta_{-}} D_{\delta}(\delta)\left(\nu^{2}-\delta^{2}\right) d \delta
\end{aligned}
$$

(with the latter forms of these equations valid for $\mu \neq 0$ ). Recall that $\nu$ must be positive. These equations for $\mu=0$ (so that $\delta=\nu$ ) show that $\mathrm{V}_{\mu}(\nu, 0)=0$ and that $\mathrm{V}_{\nu}(\nu, 0)=0$ if and only if $D_{\delta}(\nu)=\rho c^{2} \nu$. On physical grounds $D$ should be everywhere positive with $D(\delta) \rightarrow \infty$ as $\delta \rightarrow 0, \infty$. Under these conditions it is reasonable to prohibit $\mathrm{V}_{\nu}(\nu, 0)$ from vanishing by taking

$$
D_{\delta}(\delta)<\rho c^{2} \delta \text { for all } \delta>0
$$

(Here we have a supersonic behavior with respect to extensions.)

Since $\nu$ must be positive, (2.10) implies that $\mathrm{V}_{\nu}(\nu, \mu)=0$ if and only if

$$
\left[D\left(\delta_{-}\right)-D\left(\delta_{+}\right)\right]=\frac{1}{2} \rho c^{2}\left[\delta_{-}^{2}-\delta_{+}^{2}\right] .
$$

Assumption (5.20), which ensures that $\delta \mapsto D(\delta)-\frac{1}{2} \rho c^{2} \delta^{2}$ is strictly decreasing, implies that (5.21) is equivalent to $\delta_{-}=\delta_{+}$, which occurs if and only if $\mu=0$. Thus (5.20) prohibits the vanishing of $\mathrm{V}_{\mathbf{q}}$ anywhere, and therefore ensures (i) for all $\mathrm{E}$.

We can handle hypothesis (i) under weaker constitutive restrictions by restricting the range of $\mathrm{E}$ : The requirement that $\mathrm{V}_{\nu}=0$ is given by (5.21). Equations (5.19) 
and $(5.17)_{6}$, together with (2.10) and (2.23), imply that the restrictions $\vee_{\mu}=0$ and $\mathrm{V}=\mathrm{E}$ have the form

$$
\begin{gathered}
\int_{\delta_{+}}^{\delta_{-}} \delta^{2} D_{\delta}(\delta) d \delta=\nu^{2}\left[D\left(\delta_{-}\right)-D\left(\delta_{+}\right)\right]=\frac{1}{2}\left(\delta_{-}^{2}+\delta_{+}^{2}\right)\left[D\left(\delta_{-}\right)-D\left(\delta_{+}\right)\right] \\
\int_{\delta_{+}}^{\delta_{-}} \delta D(\delta) d \delta-\frac{1}{8} \rho c^{2}\left(\delta_{-}^{4}-\delta_{+}^{4}\right)=\mu \mathrm{E}=\frac{1}{4 h}\left(\delta_{-}^{2}-\delta_{+}^{2}\right) \mathrm{E} .
\end{gathered}
$$

Equation (5.22) can be written as

$$
\begin{aligned}
\frac{1}{2}\left(\delta_{-}^{2}+\delta_{+}^{2}\right)\left[D\left(\delta_{-}\right)-D\left(\delta_{+}\right)\right] & =\int_{\delta_{+}}^{\delta_{-}} \delta^{2} D_{\delta}(\delta) d \delta=\int_{\delta_{+}}^{\delta_{-}}\left\{\delta[\delta D(\delta)]_{\delta}-\delta D(\delta)\right\} d \delta \\
& =\delta_{-}^{2} D\left(\delta_{-}\right)-\delta_{+}^{2} D\left(\delta_{+}\right)-2 \int_{\delta_{+}}^{\delta_{-}} \delta D(\delta) d \delta .
\end{aligned}
$$

We substitute this equation into (5.23) to obtain

$$
\left(\delta_{-}^{2}-\delta_{+}^{2}\right)\left[D\left(\delta_{-}\right)+D\left(\delta_{+}\right)\right]=\frac{1}{2} \rho c^{2}\left(\delta_{-}^{4}-\delta_{+}^{4}\right)+\left(\delta_{-}^{2}-\delta_{+}^{2}\right) \frac{\mathrm{E}}{h} .
$$

If $\delta_{-} \neq \delta_{+}$(i.e., if $\mu \neq 0$ ), then we can cancel $\delta_{-}^{2}-\delta_{+}^{2}$ from (5.25). From the resulting equation and from (5.21) we obtain

$$
D\left(\delta_{ \pm}\right)-\frac{1}{2} \rho c^{2} \delta_{ \pm}^{2}=\frac{\mathrm{E}}{2 h} .
$$

Again we find that if (5.20) holds, then each of these equations has a unique solution, and these solutions must be the same, implying that the contradiction that $\mu=0$. Let us adopt the weaker restriction that

$$
\lim _{\delta \rightarrow \infty} \frac{D(\delta)}{\frac{1}{2} \rho c^{2} \delta^{2}} \rightarrow 0 \quad \text { as } \quad \delta \rightarrow \infty .
$$

In this case, (5.26) implies that $\delta_{-}=\delta_{+}$for sufficiently large $\mathrm{E}$, with the same contradiction.

Thus, when (5.27) holds, the gradient of $\bigvee$ can vanish only where $\mu=0$. As we observed in the paragraph containing (5.20), the gradient vanishes in this case only when $\nu$ satisfies $D_{\delta}(\nu)=\rho c^{2} \nu$. We can prohibit this from happening on $\partial \mathcal{V}(\mathrm{E})$ by simply choosing $\mathrm{E}$ such that the values of $\nu$, if any, satisfying $\mathrm{E}=2 h\left[D(\nu)-\frac{1}{2} \rho c^{2} \nu^{2}\right]$ (see $\left.(5.17)_{3}\right)$ do not satisfy $D_{\delta}(\nu)=\rho c^{2} \nu$.

The modification of assumption (5.9) so that the potential energy approaches $\infty$ merely as $\delta_{ \pm} \rightarrow 0$ ensures hypothesis (ii). We turn to hypothesis (iii). Let us assume that $G_{\gamma}(0)=0$, which follows from the assumption that the reference configuration is natural (stress-free), and assume without loss of generality that $G(0)=0$. Under the assumption that $\rho c^{2}-\Psi_{\gamma \gamma} \equiv \rho c^{2}-G_{\gamma \gamma}$ is everywhere positive, (3.6) has at most one solution for $\left(\eta, \nu^{\prime}, \mu^{\prime}\right)$. Then for $(H, \bar{N}, \bar{M})=(0,0,0),(3.6)$ has the unique solution $\left(\eta, \nu^{\prime}, \mu^{\prime}\right)=(0,0,0)$, so that $\gamma=0$, and $(5.15)_{1}$ holds.

From (3.11) and (3.6) with $H=0$ we obtain

$$
\begin{gathered}
\mathbf{p} \cdot \mathrm{K}_{\mathbf{p}}(\mathbf{q}, \mathbf{p}) \equiv \bar{N} \mathrm{~K}_{\bar{N}}+\bar{M} \mathrm{~K}_{\bar{M}}=\bar{N} \nu^{\sharp}+\bar{M} \mu^{\sharp}=\eta \Lambda_{\eta}+\nu^{\prime} \Lambda_{\nu^{\prime}}+\mu^{\prime} \Lambda_{\mu^{\prime}} \\
=\int_{-h}^{h}\left(\rho c^{2} \gamma-G_{\gamma}\right)\left(\eta+\beta_{\nu} \nu^{\prime}+\beta_{\mu} \mu^{\prime}\right) d \xi=\int_{-h}^{h}\left(\rho c^{2} \gamma-G_{\gamma}\right) \gamma d \xi .
\end{gathered}
$$


Since $G_{\gamma}(0)$ is assumed to be 0 , condition (5.7) ensures that the integrand of (5.28) is positive for $\gamma \neq 0$, so that $(5.15)_{2}$ holds.

To verify hypothesis (iv), we note that if $G_{\gamma \gamma}+C<\rho c^{2}$, then $\left(\gamma G_{\gamma}-G\right)_{\gamma}+C \gamma \leq$ $\rho c^{2} \gamma$ for $\gamma>0$, and $\mathrm{K} \geq C \int_{-h}^{h} \gamma^{2} d \xi$. Arguments like those surrounding (5.11) and (5.12) imply that hypothesis (iv) holds.

In view of these results, Theorem 5.14 implies

5.29. Theorem. Let (5.5)-(5.9), (5.20) hold with $G_{\gamma}(0)=0, G(0)=0$. Then for any $E$, Hamilton's equations (3.11) have a nonconstant periodic solution satisfying $\Lambda^{*}=E$. If the restriction (5.20) is replaced by the weaker condition (5.27), and if the limit (5.9) holds merely as $\delta_{ \pm} \rightarrow 0$, then the conclusion holds for sufficiently large $E$.

6. Conclusion. The equations for travelling waves in nonlinearly elastic rods form a system of ordinary differential equations, which can be characterized as the Euler-Lagrange equations for a Lagrangian (action) functional. These equations, however, have a mathematical structure quite different from that for Lagrange's equations of motion for a system of particles and rigid bodies, this difference signalled by the difference in the forms of the kinetic and potential energies. A major distinction is that our equations of motion cannot in general be cast into Hamiltonian form. The source of this difficulty is that our equations describe wave propagation. At the characteristic speeds, our governing equations cannot be solved uniquely for the terms with highest derivatives. We have avoided this difficulty in the preceding section by limiting our attention to waves that are either sub- or supersonic.

For the totally integrable system for travelling waves in compressible nonlinearly elastic rods, this issue was confronted head-on in [3] by allowing the integrals to have multi-valued inverses. Then travelling waves could be characterized as the level curves of a multi-valued energy over a certain phase plane. At characteristic speeds, different level curves have tangencies, at which a trajectory could switch from one branch to another, with the consequence that there could be an uncountable family of travelling waves at such a characteristic speed.

The existence of travelling waves in a neighborhood of a equilibrium state, especially a straight equilibrium state, or in a neighborhood of a purely longitudinal motion (like those of $[5,16]$, could be treated by means of bifurcation theory. (See [8] and the references cited therein.) For such purposes the symmetry results given in Section 2 would be most useful. These same results could also be used for refined global theorems (see [14]).

In Section 3 we used a global implicit function theorem to construct the Hamiltonian. Clearly, much of our development would go through without such a global theorem provided that we could find suitable bounds, say energy bounds, on the solutions of (3.3).

The growth assumptions we have made to ensure that our theorems in Section 5 hold are compatible with the assumptions made in [1] which support a collection of a priori bounds on the solutions of the full partial differential equations.

We had to go through some contortions to put our system into a suitable Hamiltonian forms. This remark suggests that the analysis of travelling waves might be more naturally conducted in the original Lagrangian formulation.

We have just treated a few special cases in which the available theory for Hamiltonian systems can applied with a reasonable effort. We have left untouched the treatment for elastic materials satisfying other constitutive restrictions. We have 
not treated the possibility of homoclinic and heteroclinic solutions of the governing equations, which correspond to pulses and bores.

The methods we have used can be applied to other problems for nonlinearly elastic rods, e.g., to the spatial motion of incompressible rods, and to rods with a structure more complicated than that of [3].

Acknowledgment. The research reported here was supported in part by a grant from the NSF.

\section{REFERENCES}

[1] S. S. Antman, Regularity properties of planar motions of incompressible rods, Disc. Cont. Dyn. Systems B, 3 (2003), pp. 481-494.

[2] S. S. Antman, Nonlinear Problems of Elasticity, 2nd edn., Springer, 2005.

[3] S. S. Antman and T.-P. Liu, Travelling waves in hyperelastic rods, Quart. Appl. Math., 36 (1979), pp. 377-399.

[4] S. S. Antman And F. Schuricht, Incompressibility in rod and shell theories, Math. Modelling Num. Anal., 33 (1999), pp. 289-304.

[5] H.-H. DAI, Exact travelling-wave solutions of an integrable equation arising in hyperelastic rods, Wave Motion, 28 (1998), pp. 367-381.

[6] I. Ekeland, Convexity Methods in Hamiltonian Mechanics, Springer, 1990.

[7] H. Hofer and E. Zehnder, Periodic solutions on hypersurfaces and a result by C. Viterbo, Invent. Math., 90 (1987), pp. 1-9.

[8] Y. A. Kuznetsov, Elements of Applied Bifurcation Theory, 3rd edn., Springer, 2004.

[9] J. D. Logan, Invariant Variational Principles, Academic Pr., 1977.

[10] J. Mawhin and M. Willem, Critical Point Theory and Hamiltonian Systems, Springer, 1989.

[11] P. H. Rabinowitz, Periodic solutions of a Hamiltonian system on a prescribed energy surface, J. Diff. Eqs., 33 (1979), pp. 336-352.

[12] P. H. Rabinowitz, On a Theorem of Hofer and Zehnder, in Periodic Solutions of Hamiltonian Systems and Related Topics, edited by P. H. Rabinowitz et al., NATO ASI Ser. C, Reidel, 1987.

[13] P. H. Rabinowitz, The prescribed energy problem for periodic solutions of Hamiltonian systems, Contemp. Math., 81 (1988), pp. 183-191.

[14] P. H. Rabinowitz, Variational methods for Hamiltonian systems, in Handbook of Dynamical Systems, Vol. 1A, edited by B. Hasselblatt and A. Katok, North Holland, 2002, pp. 10911127.

[15] H. SeIfert, Periodische Bewegung mechanischen Systeme, Math. Z., 51 (1948), pp. 197-216.

[16] T. Wright, Nonlinear waves in rods: results for incompressible elastic materials, Stud. Appl. Math., 72 (1984), pp. 149-160. 